\title{
Diffusion entropy and waiting time statistics of hard-x-ray solar flares
}

\author{
Paolo Grigolini, ${ }^{1,2,3}$ Deborah Leddon, ${ }^{1}$ and Nicola Scafetta ${ }^{1}$ \\ ${ }^{1}$ Center for Nonlinear Science, University of North Texas, P.O. Box 305370, Denton, Texas 76203 \\ ${ }^{2}$ Dipartimento di Fisica dell'Università di Pisa, Piazza Torricelli 2, 56127 Pisa, Italy \\ ${ }^{3}$ Istituto di Biofisica del Consiglio Nazionale delle Ricerche, Via San Lorenzo 26, 56127 Pisa, Italy
}

(Received 14 August 2001; published 25 March 2002)

\begin{abstract}
We show at work a technique of scaling detection based on evaluating the Shannon entropy of the diffusion process obtained by converting the time series under study into trajectories. This method, called diffusion entropy, affords information that cannot be derived from the direct evaluation of waiting times. We apply this method to the analysis of the distribution of time distance $\tau$ between two nearest-neighbor solar flares. This traditional part of the analysis is based on the direct evaluation of the distribution function $\psi(\tau)$, or of the probability $\Psi(\tau)$, that no time distance smaller than a given $\tau$ is found. We adopt the paradigm of the inverse power-law behavior, and we focus on the determination of the inverse power index $\mu$, without ruling out different asymptotic properties that might be revealed, at larger scales, with the help of richer statistics. We then use the DE method, with three different walking rules, and we focus on the regime of transition to scaling. This regime of transition and the value of the scaling parameter itself, $\delta$, depends on the walking rule adopted, a property of interest to shed light on the slow process of transition from dynamics to thermodynamics often occurring under anomalous statistical conditions. With the first two rules the transition regime occurs throughout a large time interval, and the information contained in the time series is transmitted, to a great extent, to it, as well as to the scaling regime. By using the third rule, on the contrary, the same information is essentially conveyed to the scaling regime, which, in fact, emerges very quickly after a fast transition process. We show that the DE method not only causes to emerge the long-range correlation with a given $\mu<3$, and so a basin of attraction different from the ordinary Gaussian one, but it also reveals the presence of memory effects induced by the time dependence of the solar flare rate. When this memory is annihilated by shuffling, the scaling parameter $\delta$ is shown to fit the theoretically expected function of $\mu$. All this leads us to the compelling conclusion that $\mu=2.138 \pm 0.01$.
\end{abstract}

DOI: 10.1103/PhysRevE.65.046203

\section{INTRODUCTION}

The study of solar flares is becoming popular among the researchers working at the frontier of statistical mechanics, due to the widely shared conviction that they are a signature of a significant departure from the condition of ordinary Brownian motion [1-4]. As pointed out by Wheatland [5], the distribution of times between flares, gives information on how to model flare statistics. In this paper we shall be referring to these times, denoted by us with the symbol $\tau$, as the time distance between two consecutive events, and the corresponding distribution density will be denoted by $\psi(\tau)$. Although the agreement on the fact that flare statistics depart from ordinary statistical mechanics is general, there seems to be the still unsettled issue of what is the proper model that will account for this form of anomalous statistics. Does this form of statistics reflect self-organized criticality or turbulence [3]? We think that the settlement of this delicate issue is made difficult by the fact that, although many authors claim that $\psi(\tau)$ is an inverse power law with power index $\mu$, the actual value of $\mu$ still seems to be uncertain. In fact, the authors of Ref. [1] propose $\mu=1.7$ and those of Ref. [2] claim that $\mu=2$ is the proper power-law index. Boffetta et al. [3] propose $\mu=2.4$. Finally, Wheatland explains the origin of the power-law behavior with a model yielding $\mu=3.0$ [5].

As will be made clear by the theoretical analysis of this paper, it is possible to prove, without taking a position on the origin of the inverse power-law behavior, that $\mu=3$ and
PACS number(s): 05.40.-a, 05.45.Tp, 96.60.Rd, 89.90.+n

$\mu=2$ are critical values. In fact, we note that moving from $\mu>3$ to $\mu<3$ is equivalent to a phase transition from the Gaussian to the Lévy basin of attraction [6], and moving from $\mu>2$ to $\mu<2$ implies a transition from the condition of Lévy statistics to a form of out of the equilibrium regime [7]. Thus, an uncertainty larger than the distance of the border $\mu=2$ from the border $\mu=3$ is judged by us to be an unsatisfactory condition that might delay the settlement of the issues concerning the complex dynamics underlying the waiting-time statistics. The main purpose of this paper is to illustrate a statistical method of analysis that yields a reliable value for the power index $\mu$. We hope that this result might be useful for the researchers in this interesting field of investigation and at the same time might be beneficial, in general, for all those who are interested in the statistical analysis of time series.

The outline of the paper is as follows. In Sec. II we review the method of diffusion entropy (DE) that will be a crucial step of the statistical analysis done in this paper. Although the method has been applied somewhere else [8-10], we will present a short review in order to make this paper as self-contained as possible. In Sec. III, we illustrate a dynamical model that, in general, results in time sequences that are statistically equivalent to those observed in real data. This model is not limited to the case of inverse power laws, but here we make the assumption that the shifted inverse power law is an ideal condition convenient for analyzing solar flares, and we study the explicit form emerging from this condition. In Sec. IV, we illustrate three walking prescrip- 
tions that will be used to convert the real data into random trajectories. The benefit of adopting several walking prescriptions was discussed in Ref. [9]. Here we introduce two additional rules and we apply both of them as well as one of those introduced in Ref. [9]. In Sec. V we prove that the numerical evaluation of the probability of getting a waiting time larger than a given $\tau$ yields a value for $\mu$ more accurate than that afforded by the waiting-time distribution $\psi(\tau)$. In Sec. VI we show how to process the data to make an efficient use of the DE method. In Sec. VII we use the DE method to further reduce the error of Sec. V. We devote Sec. VIII to concluding remarks.

\section{DIFFUSION ENTROPY}

The main idea of this approach to scaling is remarkably simple. Let us consider a sequence of $M$ numbers $\xi_{i}(t)$ with $i=1, \ldots, M$. The purpose of the DE algorithm is to establish the possible existence of a scaling, either normal or anomalous, in the most efficient way as possible without altering the data with any form of detrending. Let us select first of all an integer number $l$, fitting the condition $1 \leqslant l \leqslant M$. This integer number will be referred to as "time." For any given time $l$ we can find $M-l+1$ subsequences defined by

$$
\xi_{i}^{(s)} \equiv \xi_{i+s}, \quad s=0, \ldots, M-l .
$$

For any of these subsequences we buildup a diffusion trajectory, labeled with the index $s$, defined by the position

$$
x^{(s)}(l)=\sum_{i=1}^{l} \xi_{i}^{(s)}=\sum_{i=1}^{l} \xi_{i+s} .
$$

Let us imagine this position as referring to a Brownian particle that at regular intervals of time has been jumping forward or backward according to the prescription of the corresponding subsequence of Eq. (1). This means that the particle before reaching the position that it holds at time $l$ has been making $l$ jumps. The jump made at the $i$ th step has the intensity $\left|\xi_{i}^{(s)}\right|$ and is forward or backward according to whether the number $\xi_{i}^{(s)}$ is positive or negative.

We are now ready to evaluate the entropy of this diffusion process. To do that we have to partition the $x$ axis into cells of size $\epsilon(l)$. When this partition is made we have to label the cells. We count how many particles are found in the same cell at a given time $l$. We denote this number by $N_{i}(l)$. Then we use this number to determine the probability that a particle can be found in the $i$ th cell at time $l, p_{i}(l)$, by means of

$$
p_{i}(l) \equiv \frac{N_{i}(l)}{(M-l+1)} .
$$

At this stage the entropy of the diffusion process at time $l$ is determined and reads

$$
S_{d}(l)=-\sum_{i} p_{i}(l) \ln \left[p_{i}(l)\right]
$$

The easiest way to proceed with the choice of the cell size $\epsilon(l)$, is to assume it to be independent of $l$ and determined by a suitable fraction of the square root of the variance of the fluctuation $\xi(i)$. In the case in which the numbers $\xi_{i}$ are +1 , 0 , and $-1, \quad \epsilon=1$ is the natural choice.

Before proceeding with the illustration of how the DE method works, it is worth making a comment on how to define the trajectories. The method we are adopting is based on the idea of a moving window of size $l$ that makes the $s$ th trajectory closely correlated to the next, the $(s+1)$ th trajectory. The two trajectories have $l-1$ values in common. It is worth making a comparison with the technique of detrended fluctuation analysis (DFA) [11]. The DFA is a popular method of scaling analysis, aiming at detecting the longrange correlations in seemingly nonstationary time series that in the last few years has been used in more than 100 publications [12]. The DFA is based on nonoverlapping windows, and, consequently, trajectories with different labels are totally independent from one another. The motivation for using overlapping windows, with the DE method, is given by our wish to establish a connection with the Kolmogorov-Sinai (KS) entropy $[13,14]$. In Sec. III we shall make further comments on this connection. The KS entropy of a symbolic sequence is evaluated by moving a window of size $l$ along the sequence. Any window position corresponds to a given combination of symbols, and from the frequency of each combination it is possible to derive the Shannon entropy $S(l)$. The KS entropy is given by the asymptotic limit $\lim _{l \rightarrow \infty} S(l) / l$. We believe that the same sequence, analyzed with the DE method, at the large values of $l$ where a finite KS entropy shows up, must yield a well-defined scaling $\delta$. To realize this correspondence we carry out the determination of the DE by using the same criterion of overlapping windows as that behind the KS entropy.

Details on how to deal with the transition from the shorttime regime, sensitive to the discrete nature of the process under study, to the long-time limit where both space and time can be perceived as continuous, are given in Ref. [10]. Here we make the simplifying assumption of considering large enough times as to make the continuous assumption valid. In this case, the trajectories, built up with the above-illustrated procedure, correspond to the following equation of motion:

$$
\frac{d x}{d t}=\xi(t)
$$

where $\xi(t)$ denotes the value that the time series under study gets at the $l$ th site of the sequence under study. This means that the time $t \equiv l$ (with $l \gg 1$ ) is thought of as a continuous and that the function $\xi(l)$ is a function of this continuous time. In this case the Shannon entropy reads

$$
S(t)=-\int_{-\infty}^{\infty} d x p(x, t) \ln [p(x, t)] .
$$

We also assume that

$$
p(x, t)=\frac{1}{t^{\delta(t)}} F\left(\frac{x}{t^{\delta(t)}}\right)
$$


and that $F(y)$ maintains its form, namely that the statistics of the process is independent of time. Let us plug Eq. (7) into Eq. (6). Using a simple algebra, we get

$$
S(\tau)=A+\delta(\tau) \tau,
$$

where

$$
A \equiv-\int_{-\infty}^{\infty} d y F(y) \ln [F(y)]
$$

and

$$
\tau \equiv \ln (t) .
$$

The assumptions made to get the result of Eq. (8) are not correct during the transition process, and consequently the DE method can be used as a reliable way to detect scaling only in the long-time limit. The DE can be used, however, to shed light into the regime of transition that is deeply connected with the foundation itself of statistical mechanics. According to Khinchin [15] the central limit theorem is fundamental for the realization of canonical equilibrium. As well known, a process resulting from the sum of $N$ independent variables yields a Gaussian distribution, provided that $N$ is large and the single variables have a probability distribution with a finite second moment. A physical process making $N$ increase from values of the order of unity to values so large as to fit the prediction of the central limit theorem can be perceived as a transition from the microscopic to the macroscopic regime, where thermodynamics applies. If the microscopic variables do not have a finite second moment, the ordinary central limit theorem must be replaced by the generalized central limit theorem [16] and in the limiting case of $N \rightarrow \infty$ we find Lévy rather than Gauss statistics. We can generalize the point of view of Khinchin and consider also in this case the process of transition of $N$ from small to large values as a form of transition from the microscopic to the thermodynamic regime.

Due to the nature of the DE method, the role of $N$ is here played by the "time" $t$. The microscopic regime refers to the fluctuation of $\xi_{i}$ and the macroscopic regime corresponds to the fluctuations of the diffusion coordinate $x(t)$. The time evolution of $\delta(t)$ towards the final value, independent of time, reflects the transition from dynamics to thermodynamics.

We shall adopt three different walking rules (see Sec. IV). The first two rules are characterized by an extended regime of transition from dynamics to thermodynamics. Notice that the real data available are finite, thereby producing saturation effects in the long-time regime. Consequently, the region where the ideal scaling shows up, is an intermediate time region following the extended initial transition and preceding the long-time saturation regime. This has the effect of reducing the size of the time region that can be fruitfully used for scaling detection. As we shall see, this is the reason why the DE method must be supplemented by the use of artificial sequences. The third rule, on the contrary, yields a fast transition to the thermodynamic regime, thereby allowing us to determine the scaling by a direct use of Eq. (8), with $\delta(t)$ assuming the time independent value of the thermodynamic limit.

\section{DYNAMIC MODEL}

The solar flares analyzed in this paper are perceived as a sequence of events occurring at unpredictable times $t_{i}$, with $i=1, \ldots, M$, where $M$ is the label of the last event considered. We do not take into account the intensity of these events, which will be studied somewhere else. Thus, the most important property for us to study, is the time distribution density $\psi(\tau)$, with $\tau$ denoting the time distance between two nearest-neighbor events $\tau_{i} \equiv t_{i+1}-t_{i}$. Let us make the assumption that the experimental analysis of the time series yields the form

$$
\psi(\tau)=(\mu-1) \frac{T^{\mu-1}}{(T+\tau)^{\mu}} .
$$

We make the key assumption that the numbers $\tau_{i}$ are uncorrelated. As we shall see, the theory of this paper affords also a criterion to assess if this crucial assumption is correct or not. Under this key assumption we can buildup a dynamic model that is statistically equivalent to the solar dynamics generating the sequence of the $t_{i}$ 's. Let us consider the dynamic process,

$$
d y / d t=\lambda y^{z},
$$

with $z>1$. Let us imagine that the trajectory $y(t)$ moves within the interval $[0,1]$. Let us assume also that when the trajectory reaches the right border of this interval it is injected back within this interval by means of a random selection of the initial position $y(0)$. The random selection is done by using a random number generator that assigns the same probability to the numbers of the interval $[0,1]$. The connection between the initial condition and the exit time $\tau$ is given by

$$
y(0)=[1+(z-1) \lambda \tau]^{-(1) /(z-1)} .
$$

This leads immediately to the distribution of Eq. (11) with

$$
\mu=\frac{z}{z-1}
$$

and

$$
T=\frac{\mu-1}{\lambda} .
$$

We are now equipped to establish a connection with the entropy production per unit of time. Randomness here is involved at the moment of selecting the initial condition, and is characterized by an unknown amount of entropy increase, $H$. If $\mu>2$, the distribution of Eq. (11) yields a finite mean waiting time, 


$$
\langle\tau\rangle=\frac{T}{\mu-2} .
$$

It is evident then that the rate of entropy production per unit of time is given by

$$
h_{E}=H \frac{(\mu-2)}{T} \text {. }
$$

Using the dynamic model it is possible to establish a more proper connection with the KS entropy. However, this is of no great relevance within the context of the present paper. Therefore, we limit ourselves to considering the entropy production of Eq. (17), where the subscript $E$ stands for "external." In fact, in the picture adopted in the present paper the source of entropy production is the random selection of the numbers of the interval $[0,1]$, an action external to the process under study. It has to be pointed out that this external entropy production is subtly related to the KS entropy, which, on the contrary, is interpreted as being of internal origin [17]. This is so because the dynamical model is a map with a very sharp chaotic region that reduces to a set of zero measure, confined to the point $y=1$, in the limiting condition where the idealized model of this section applies.

In the case $\mu<2$ the entropy produced is proven [7] to be the following function of time:

$$
S(t) \propto t^{\mu-1}
$$

It is evident that in the limiting case of very large time values the entropy production per unit of time vanishes, thereby implying that the condition, $\mu=2$ is a border at which a kind of phase transition occurs. In the region $\mu>2$ the dynamical system of Eq. (12) has an invariant distribution. In the region $\mu<2$ the system does not have an invariant distribution [7]. From an intuitive point of view we can imagine that during the observation process the system keeps moving towards an equilibrium distribution, as a kind of Dirac $\delta$ function located at $y=0[7]$. The time necessary to reach this invariant distribution is infinite.

In conclusion, an infinitesimally small change from $\mu$ $>2$ to $\mu<2$ would have the effect of annihilating the invariant distribution and of making the process "nonstationary." The method of analysis of this paper will allow us to assess that $\mu=2.138 \pm 0.01$, namely, that the solar flares fluctuations are stationary, even if very close to the border with the "nonstationary" region. This result will be obtained by a direct evaluation of $\mu$, supplemented by the adoption of the DE method. As we shall see, this conclusion is reached after settling a major problem caused by the existence of a genuine form of nonstationary behavior; this kind of nonstationary behavior means that the dynamic rules behind the process under study change upon change of time. This will lead us to the final conclusion that the model of Eq. (12) is a fairly accurate way of mimicking solar flare dynamics with $z<2(\mu>2)$. In Secs. VII and VIII we shall make some conjectures on how to improve this model to take into account the time dependence of the solar flare rate.

\section{ON THREE DISTINCT PRESCRIPTIONS TO WALK}

The scaling detected by the DE method is not independent of the walking rules that we adopt. The outcomes of DE method are not unique, due to the dependence of the scaling parameter $\delta$ on the walking rules, and this casts doubts on this method of analysis. However, the task of this analysis is an indirect evaluation of the waiting-time distribution $\psi(\tau)$, or, equivalently, in the inverse power-law case, of the index $\mu$. If we take for granted the inverse power-law structure of $\psi(\tau)$, the power index $\mu$ is unique. We adopt the following prescriptions for the random walker:

(1) Make a jump of fixed intensity, only when you meet an event, and do it always in the same direction.

(2) As with Rule (1) make a jump only when you meet an event, but do it either in the positive or negative direction according to a coin tossing prescription.

(3) Walk at fixed interval of times, with jumps in the same direction, of intensity proportional to the time distance between two nearest-neighbor events.

Note that here we analyze the sequence $\left\{\tau_{i}\right\}$, where each value $\tau_{i}$ denotes the time distance between two nearestneighbor flares (regarded as events). Thus, Rules (1) and (2) imply that the random walker makes instantaneous jumps at the times of flare occurrence. With Rule (3) the random walker, at times $t=1,2, \ldots, n, \ldots$, makes jumps ahead of intensity equal to the values $\tau_{i}$ of the sequence under study. Note that Rule (1) is one of the two rules used in Ref. [9]. Here we use Rules (2) and (3). Using the theory of Ref. [9], which, in turn, essentially rests on the generalized central limit theorem [16] and on the work of Feller [18], we obtain the following prescriptions:

$$
\begin{aligned}
& \delta=\left\{\begin{array}{l}
\mu-1, \quad 1<\mu<2 \\
1 /(\mu-1), \quad 2<\mu<3 \\
0.5, \quad \mu>3,
\end{array}\right. \\
& \delta=\left\{\begin{array}{l}
0.5(\mu-1), \quad 1<\mu<2 \\
0.5, \quad \mu>2,
\end{array}\right.
\end{aligned}
$$

and

$$
\delta=1 /(\mu-1), \quad \mu>1,
$$

for Rules (1), (2), and (3), respectively.

Figure 1 shows clearly that the adoption of Rule (1) alone would yield two distinct possible values for $\mu$ when $\delta$ gets values within the interval $[0.5,1]$. However, the joint adoption of this and the other two rules settles this ambiguity. We also notice that both Rule (1) and Rule (2) reflect the phasetransition character of the condition $\mu=2$, while Rule (3), apparently, does not. However, we see that Rule (3) for $\mu$ $<2$ yields a value of $\delta>1$, namely, a diffusion process faster than the ballistic diffusion. This is a consequence of the nonstationary nature of the condition $\mu<2$.

It is important to stress that these rules imply that the numbers $\tau_{i}$ are not correlated. Furthermore, these rules rest on the assumption that the asymptotic limit of $\psi(\tau)$ is an 


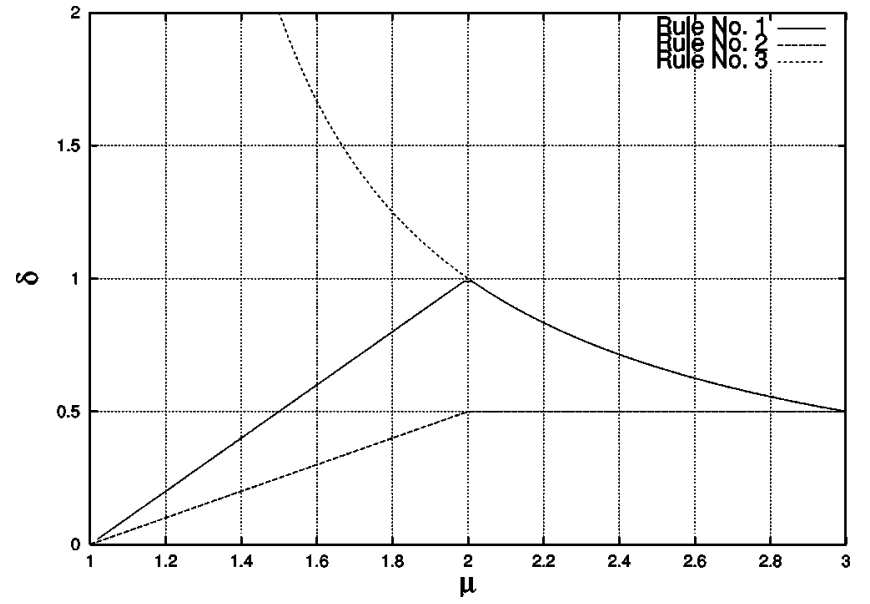

FIG. 1. $\delta$ as a function of $\mu$ according to three rules. The solid, dashed, and dotted lines denote Rules (1), (2), and (3), respectively.

inverse power-law distribution with no truncation. We shall see that the DE method is sensitive to the correlation among the numbers $\tau_{i}$, and that the 11-year solar cycle is responsible for that correlation. As to the truncation of the inverse power law at the large distances, this is another delicate issue worth of some comments. Laherrére and Sornette [19], suggest that the stretched exponential family might have a theoretical motivation stronger than the power-law distribution. On the other hand, in the intermediate time region a stretched exponential is indistinguishable from a power law. The two proposed fitting functions become distinguishable one from the other in the long-time regime, which is affected by poor statistics. However, the work of Refs. [20] and [21] show that a truncation of the power law of $\psi(\tau)$ at large times yield an ultraslow convergence to normal diffusion, with effects that are beyond the range of observation of the DE analysis, due to the data statistical limitation.

We shall see that both Rule (1) and Rule (2) yield a very slow transition to the scaling regime. Due to the statistical limitation of our data, the scaling regime turns out to be a relatively short-time region between transition and saturation regime. Thus, we shall be forced to carry out our analysis with the help of artificial sequences with the same number of terms as the real data, by fitting the DE curves produced by the real data with the DE curves generated by the artificial sequences. The adoption of the third rule, on the contrary, yield a fast transition to the thermodynamic regime and, consequently, allows us to determine directly the value of $\delta$. In both cases, however, the physical consequences of a possible truncation of the inverse power law are beyond our range of observation.

\section{STATISTICAL ANALYSIS OF THE REAL DATA: $\psi(\tau)$ AND $\Psi(\tau)$}

In this section we plan to derive the waiting-time distribution $\psi(\tau)$ directly from the statistical analysis of the real data, the $\mathrm{x}$ rays emitted by solar flares in the case here under study. At first sight, one might think that a direct determination of $\psi(\tau)$ is more convenient than any indirect approach.

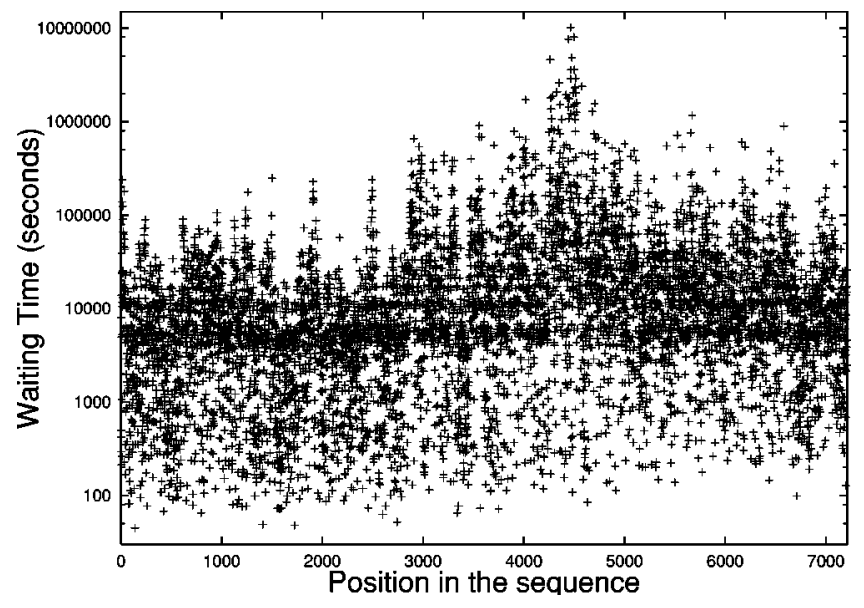

FIG. 2. The original sequence of the solar flares, waiting times. Note the logarithmic scale of ordinates.

Actually, it is not so. As mentioned in Sec. I, we find that the evaluation of the probability of finding no time distance larger than a given $\tau$, denoted by $\Psi(\tau)$, defined by

$$
\Psi(\tau) \equiv \int_{\tau}^{\infty} \psi(t) d t
$$

is more convenient than the direct evaluation of $\psi(\tau)$. In later sections we shall prove a striking property: the evaluation of $\mu$ through the DE method, an approach less direct than the evaluation of $\Psi(\tau)$, is still more efficient.

The data are a set of 7212 hard $\mathrm{x}$-ray peak flaring event times obtained from the BATSE/CGRO (Burst and Transient Source Experiment aboard the Compton Gamma Ray observatory satellite) solar flare catalog list. The data is a 9-year series of events from 1991 to 2000. If the time $\Delta t$ between two consecutive solar flares is expressed in seconds, the range goes from 45 to $10000000 \mathrm{sec}$, as shown in Fig. 2. Figure 3 shows the rate of solar flares per month from April 1991 to May 2000. The set of data studied here concerns a time period of 9 years, and, consequently, a large part of the whole 11-year solar cycle. Figure 3 shows that during a large

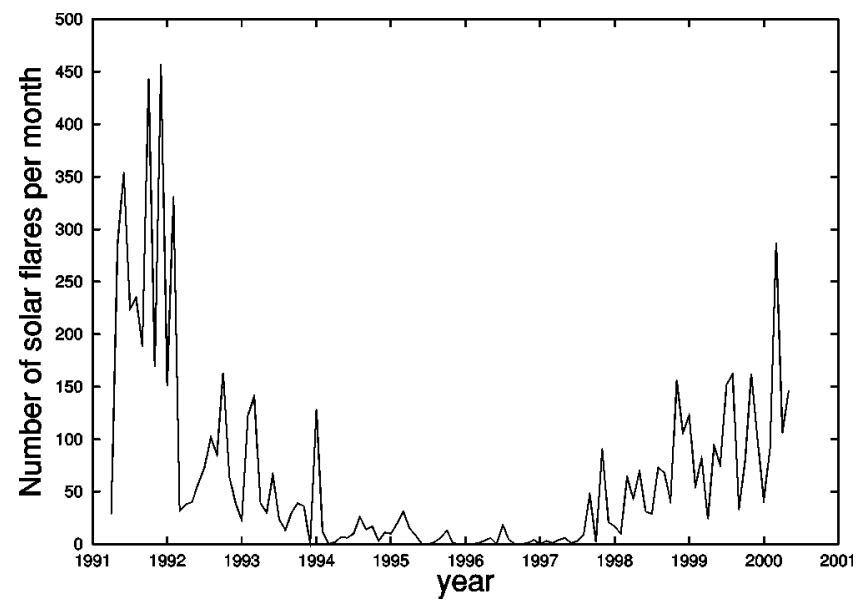

FIG. 3. Number of solar flares per month from April 1991 to May 2000. 


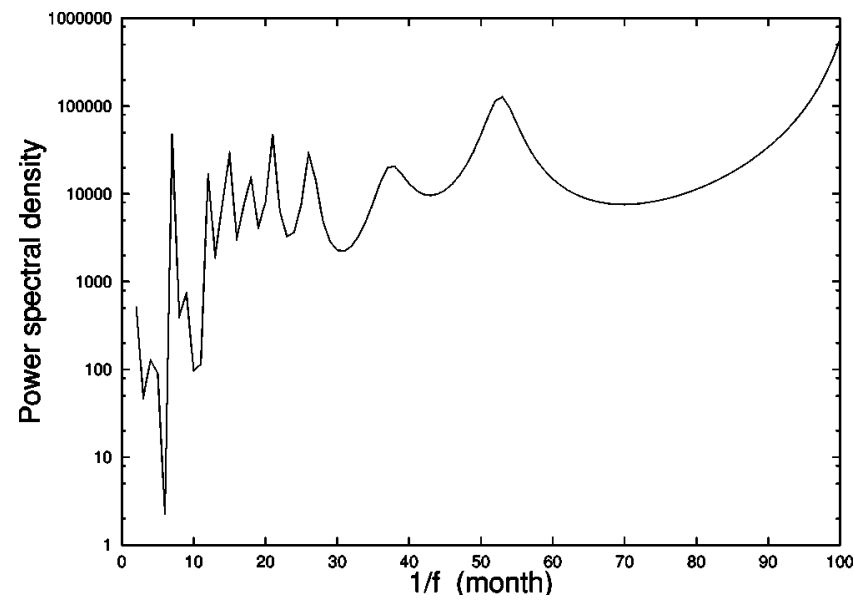

FIG. 4. The solid curve was obtained by using the maximum entropy method [22].

portion of this 11-year cycle the flare rate undergoes big changes, thereby significantly departing from the uniform distribution. Furthermore, it is worth remarking that, as shown by Fig. 4, the 11-year solar cycle is not a mere harmonic oscillation with the period of 11 years, but a complex dynamic process with many components.

The direct evaluation of the waiting-time distribution $\psi(\tau)$, needs the data to be distributed over many bins with the same size. When only a few data are available, the bin size cannot be too small, and, in turn, the adoption of bins of large size can produce incorrect power-law indices. In proceeding with the direct evaluation of the key parameter $\mu$, first of all, we have to adopt a proper criterion to determine the size $\Delta_{i}$ of the $i$ th bin. We note that the waiting-time distribution is expected to be an inverse power law. If we adopted bins of equal size, those corresponding to large times would collect a very limited amount of data, thereby resulting in a nonreliable evaluation of the frequencies. To bypass this difficulty we adopt bin sizes that are constant in the logarithmic scale. This means that $\ln \left(\tau_{i}\right)-\ln \left(\tau_{i-1}\right)$, where $\tau_{i}$ and $\tau_{i-1}$ are the middle times of two consecutive bins, is constant. We define the width of the $i$ th bin as $\Delta$ $=\tau_{i}-\tau_{i-1}$, thereby making it become an exponentially increasing function of the sequence position, so as to widely compensate for the density decrease. In this representation the probability density $\psi\left(\tau_{i}\right)$ is expressed by

$$
\psi\left(\tau_{i}\right)=\frac{N_{i}}{N \Delta_{i}}
$$

where $N$ is the total number of data points, $N_{i}$ is number of points located within the $i$ th bin, and $\Delta_{i}$, as earlier said, is the width of the $i$ th bin.

The fitting is done by using the prescription of a power law of the type of Eq. (11),

$$
\psi(\tau)=\frac{A_{1}}{(T+\tau)^{\mu}}
$$

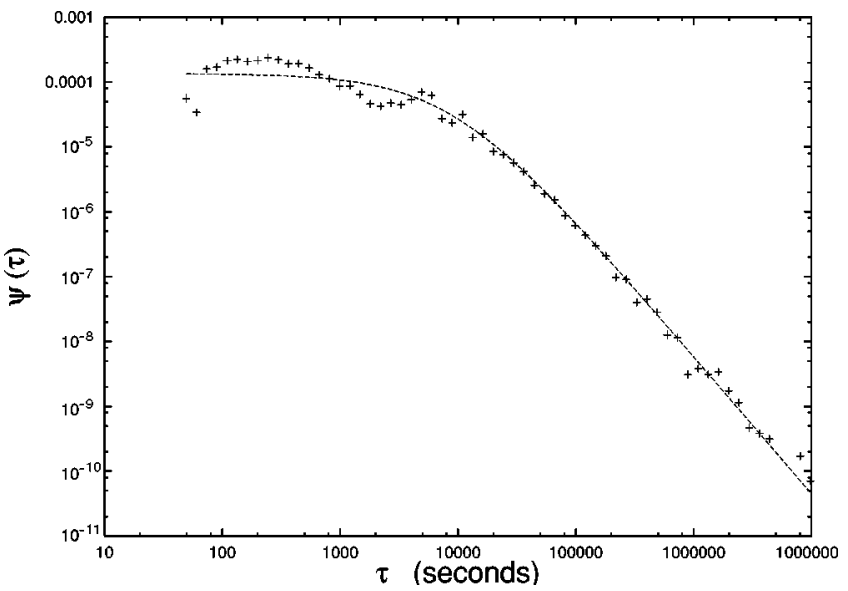

FIG. 5. The waiting-time distribution $\psi(\tau)$ as a function of $\tau$. The crosses refer to real data. The dashed line is the fitting function of Eq. (24) with $A_{1}=31006, T=8787$, and $\mu=2.12$.

with $A_{1}, T$, and $\mu$ being three independent fitting parameters. It is worth noting that the normalization condition reduces the three independent parameters to two, as made clear by Eq. (11), which is a function of only $\mathrm{T}$ and $\mu$. We find it to be more convenient to adopt three independent fitting parameters, with the understood proviso that the departure of $A_{1}$ from the value $(\mu-1) T^{\mu-1}$ can be interpreted as a way to estimate the inaccuracy of the adopted fitting procedure.

The fitting is done by using an implementation of the nonlinear least-squares (NLLS) Marquardt-Levenberg algorithm [22]. The NLLS algorithm may not give unique values for the fitting parameters. It needs initial guesses for the free parameters and the final results may change or be affected by huge errors. This fitting procedure yields $T=8787, \mu$ $=2.12 \pm 0.32$, and $A_{1}=31006$. The evaluated value of $A_{1}$ is not far from the value 29236 that would be required by the normalization condition. However, there are very large errors of the order of $100 \%$, with an error on the parameter $\mu$ of the order of $15 \%$, thereby implying $1.80<\mu<2.44$. This means that the result of this fitting procedure would prevent us from assessing the important question raised in Sec. III on whether the process is stationary or nonstationary. The large error of this procedure depends upon the initial values assigned to the three fitting parameters $T, \mu$, and $A_{1}$, whose choice requires a more efficient criterion. It also depends on the fact that there are oscillations around the fitting curve, as clearly illustrated by Fig. 5.

As earlier mentioned several times, a more accurate fitting is obtained using the function $\Psi(\tau)$. Again we do not pay attention to the normalization constraints and we adopt the following fitting function:

$$
\Psi(\tau)=A_{2}\left(\frac{1}{T+\tau}\right)^{\mu-1}
$$

As shown by Fig. 6, the fitting of the real data is now much more accurate than that of Fig. 5. The fitting parameters used are $\quad A_{2}=30657 \pm 16590, \quad T=8422 \pm 500, \quad \mu=2.144$ \pm 0.05 . This sets on the key parameter $\mu$ the constraint $2.094<\mu<2.194$, which has the very attractive property of 


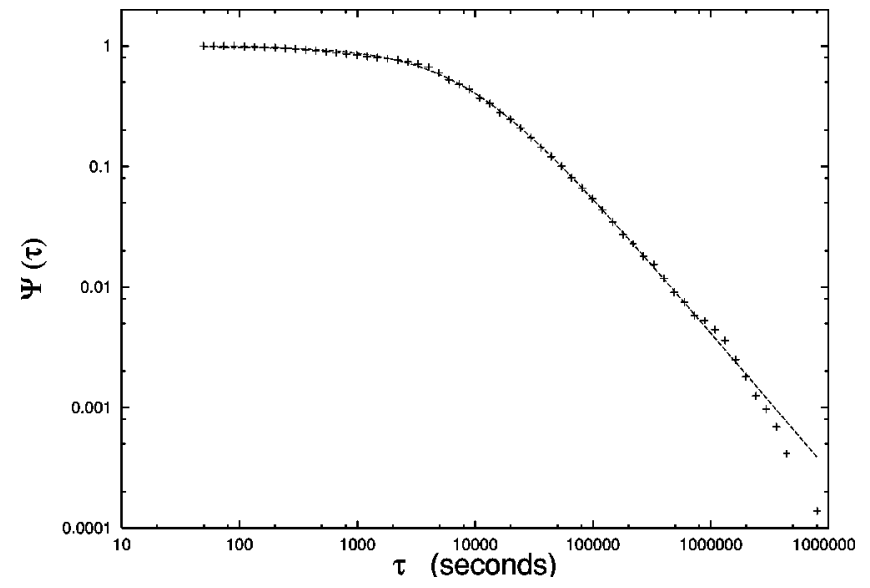

FIG. 6. $\Psi(\tau)$ as a function of $\tau$. The crosses refer to real data and the dashed line denotes the fitting function of Eq. (25) with $A_{2}=30567, T=8422$, and $\mu=2.144$.

establishing the stationary nature of the dynamic model behind the solar flares fluctuations. The results of this search for $\mu$, based on the direct evaluation of $\psi(\tau)$ and on the use of $\Psi(\tau)$, are summarized in Table I. We note that the uncertainty interval associated with the use of $\Psi(\tau)$ is contained within the wider uncertainty interval produced by the use of $\psi(\tau)$. This means that we are coming closer to the real value of $\mu$. The width of the uncertainty interval will be further reduced by using the DE method.

\section{DIFFUSION ENTROPY OF SOLAR FLARES}

This section is devoted to the analysis of the solar flares data by means of the DE method. The final result will be given by $\mu=2.138 \pm 0.01$, namely, a value for $\mu$ even more accurate than that obtained in Sec. V by using $\Psi(\tau)$. We shall prove also that the DE method allows us to establish some aspects of the dynamics behind solar flares that would be overlooked by an analysis based only on the use of the waiting-time distribution.

The first issue that we have to solve is how to process the data so as to apply the three walking rules of Sec. IV. The data accessible to us are the times $\tau_{i}=t_{i}-t_{i-1}$, with $t_{i}$ and $t_{i-1}$ denoting the time of occurrence of the $i$ th and the $(i$ $-1)$ th solar flare, respectively. However, the direct adoption of these numbers would result in technical difficulties that are bypassed by referring ourselves to the new sequence of numbers

$$
\beta_{j}=\operatorname{Int}\left[\frac{\Delta t_{j}}{\Lambda}\right]+1
$$

where $\operatorname{Int}[x]$ denotes the integer part of $x$. The adoption of $\Lambda=1$ would be virtually equivalent to referring ourselves to

TABLE I. Results of search for $\mu$.

\begin{tabular}{cc}
\hline \hline$\psi(\tau)$ & $\Psi(\tau)$ \\
\hline $1.80<\mu<2.44$ & $2.094<\mu<2.194$ \\
\hline \hline
\end{tabular}

the original sequence of numbers. However, preliminary trials with changing values of $\Lambda$ led us to conclude that there are problems with the adoption of both excessively small and excessively large values of $\Lambda$. The adoption of excessively small values of $\Lambda$ would make the computer analysis too slow and would require an excessively large amount of computer memory. This is the reason why we cannot use the original sequence of numbers. The adoption of excessively large values of $\Lambda$, on the other hand, would produce statistical saturation, and a consequent subdiffusion process that would not accurately reflect the dynamics behind the data. We adopted the criterion of using the largest value of $\Lambda$ compatible with negligible saturation effect. Preliminary attempts made it possible for us to assess that this convenient value is given by $\Lambda=3600$.

After processing the data, we have to realize the three walking rules of Sec. IV. We note that according to the prescription of Sec. II, diffusion is generated by the random walker jumping at any time step. The random walker makes jumps of intensity $\left|\xi_{i}\right|$, ahead or backward, according to whether $\xi_{i}>0$ or $\xi_{i}<0$. Thus, we create a new sequence $\xi_{i}$, of 0 's and 1's, with the following prescription. We consider a sequence of infinite empty sites, labeled by the integer index $i$, considered as a discrete time, running from $i=1$ to $i=\infty$. We divide this sequence into patches of width $\beta_{j}$. The first patch consists of the sites $i=1, \quad i=2, \ldots, i=\beta_{1}$, the second patch consists of the sites $i=\beta_{1}+1, \quad \beta_{1}+2, \ldots, \beta_{1}$ $+\beta_{2}$, and so on. We assign the value 0 to all the sites of the same patch but the last site. This means that the random walker walks only at the end of the patch, namely, at the occurrence time of an event. To apply Rule (1), with the random walker always moving in the same direction, we always assign to the last site of a given patch the value of 1 . To apply Rule (2) we assign to the last site of any patch either the value 1 or the value -1 , according to the coin tossing rule. The coin tossing prescription is realized by using a random number generator. To reduce the risk of artificial periodicity we create ten different sequences, each corresponding to a different random distribution of 1 's and -1 's. For any sequence we run the DE method and then we make the average over the ten resulting DE curves. To apply the Rule (2), which will be shown in action in Sec. VII C, we have to identify $\xi_{i}$ with $\beta_{i}$.

The DE results obtained applying Rule (1) are illustrated in Fig. 7. This figure shows one of the benefits of the DE method. According to Rule (1), we have to use the prescription of Eq. (19). The most accurate of the values of $\mu$, discussed in Sec. V, is $\mu=2.144$. This value, being smaller than 3 and larger than 2, makes us adopt the formula $\delta=1 /(\mu$ -1 ), and yields the scaling parameter $\delta=0.874$, which is the slope of the straight line of Fig. 7.

This theoretical prediction implies that the times $\tau_{i}$ of the sequence $\left\{\tau_{i}\right\}$ are not correlated with each other. In the specific case of seasonal periodicity described by harmonic oscillations, the numerical results of Ref. [8] prove that the scaling detected by the DE, as well as by other methods to detect scaling, is higher than the Brownian motion scaling $\delta=0.5$. This is so even when there is no correlation in addition to seasonal periodicity. We eliminate this effect, by shuf- 


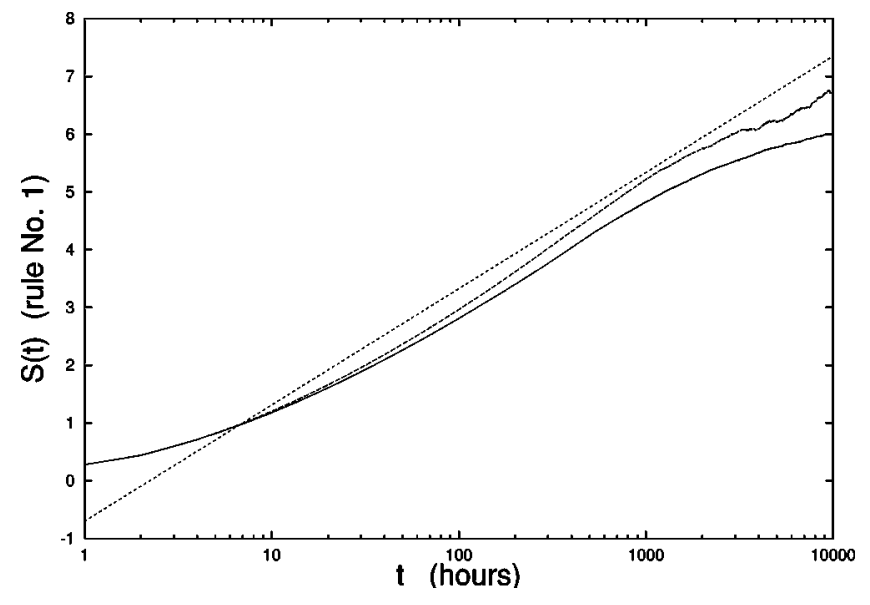

FIG. 7. DE as a function of time according to Rule (1). The dotted straight line illustrates the slope of entropy increase corresponding to $\mu=2.144$, and $\delta=0.874$, which is the best value of $\mu$ afforded by the analysis of Sec. V. The dashed line is the DE curve generated by the nonshuffled real data. The solid line is the DE curve generated by the shuffled real data.

fling the data. The DE method can be applied to both the original sequence of $\beta_{i}$ and to the shuffled sequence. If the DE yields two different curves, this is a proof of the fact that there is memory in the original sequence. This is an important property that cannot be revealed by the analysis of the waiting-time distribution $\psi(\tau)$. Figure 7 shows that this is the case. In fact we see that the DE curve corresponding to the shuffled data, after the transition region at short time and before saturation, has a slope distinctly smaller than the curve referring to the nonshuffled data. Furthermore, this slope is closer to the slope of the straight line corresponding to the finding of Sec. V, which yields $\mu=2.144$, and, consequently, according to Eq. (19), $\delta=0.874$. However, both shuffled and nonshuffled data yield saturation effects at a time scale of the order of $t_{s a t}=1500 \mathrm{~h}$. These saturation effects set limits to the accuracy of the determination of the value of $\mu$ by means of the DE method.

In Fig. 8 we illustrate the results obtained by using Rule (2). It is remarkable that in this case the shuffled data yield, with the DE method, an entropy increase faster (rather than slower) than the nonshuffled data. This is a consequence of the fact that in this case the deviation from ordinary diffusion, produced by time periodicity, would generate subdiffusion rather than superdiffusion. We notice that the difference between the shuffled and nonshuffled curves is smaller than in the case of Fig. 7 [Rule (1)] and that the saturation effects show up at later times. We thus conclude that Rule (2) is much less sensitive to periodicities and to saturation effects than Rule (1).

\section{A FURTHER IMPROVEMENT: USE OF ARTIFICIAL SEQUENCES}

We have seen that the DE method reveals the existence of memory effects that are overlooked by the direct evaluation of the waiting-time distribution. However, as pointed out in Sec. II and illustrated by the numerical results of Sec. VI, the

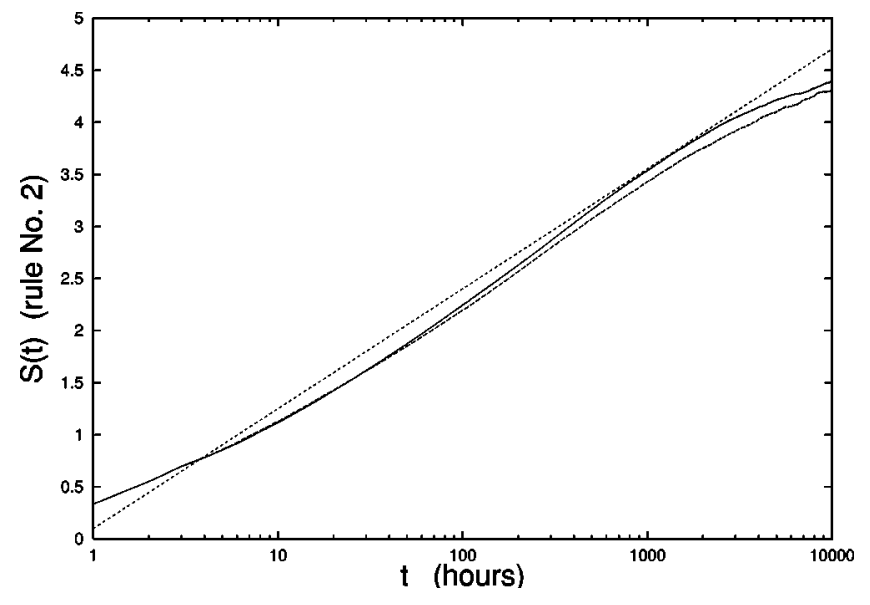

FIG. 8. DE as a function of time according to Rule (2). The dotted straight line illustrates the slope of entropy increase corresponding to $\mu=2.144, \delta=0.5$, which is the best value of $\mu$ afforded by the analysis of Sec. V. The dashed line is the DE curve generated by the nonshuffled real data. The solid line is the DE curve generated by the shuffled real data.

time region where the DE method might be fruitfully used to detect scaling, is reduced to an intermediate time region, after the transition from dynamics to thermodynamics, and before the saturation effects. This has the unwanted effect of setting limitations to the accuracy of the DE method. To bypass this difficulty we generate artificial sequences with the same statistical limitations of the real data, and then we search for the parameter $\mu$ that establishes the most accurate fitting with the DE curves derived from real data.

To make this procedure as reliable as possible we proceed as follows. We assume that $\psi(\tau)$ has the form

$$
\psi(\tau)=\frac{A}{(T+\tau)^{\mu}},
$$

where $T$ and $\mu$ are our fitting parameters. The constant $A$ is determined by the normalization condition through

$$
\frac{1}{A} \equiv \int_{45(T+\tau)^{\mu}}^{\infty} \frac{1}{\tau}
$$

The fitting parameters are made to change around the mean values established by the results of Sec. V, which yield $\mu$ $=2.144 \pm 0.05$ and $T=8422 \pm 500$. Note that in the real data no time exists with a value smaller than $\tau=45 \mathrm{sec}$. This is the reason why the integration in Eq. (28) is done from 45 to $\infty$ rather than from 0 to $\infty$. The number of data available to us are 7211. Thus we produce 7211 values of $\tau_{i}$, according to the prescription

$$
\tau_{i}=\left[\frac{1}{(T+45)^{\mu-1}}-\frac{(\mu-1) y_{i}}{A}\right]-T,
$$

with the number $y_{i}$ randomly selected in the interval $[0,1]$. It is straightforward to prove that the resulting distribution of $\tau_{i}$ is the same as that of Eq. (27) and fits the condition of Eq. 


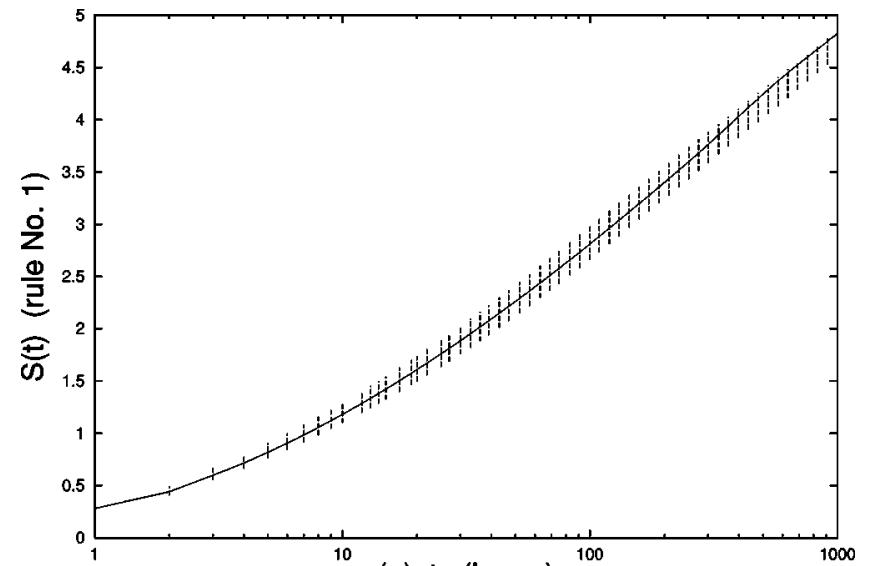

(a) $t$ (hours)

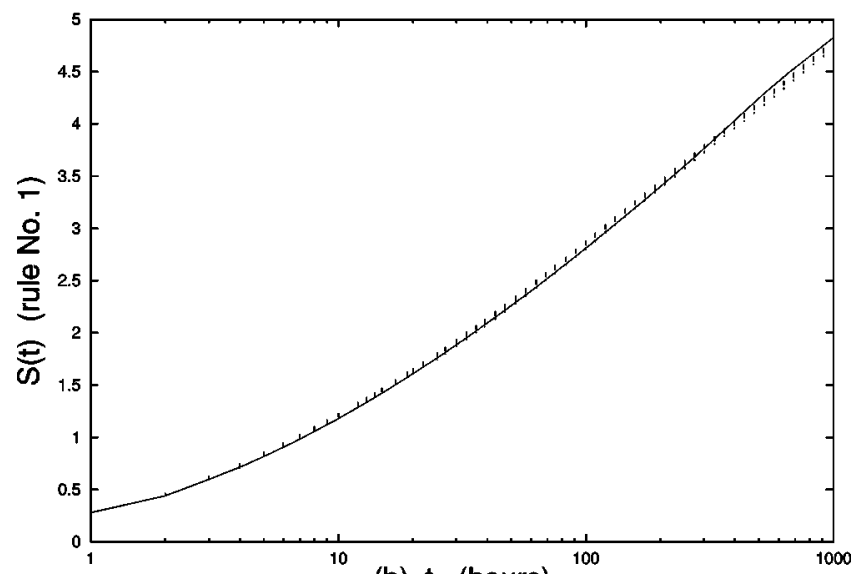

(b) $t$ (hours)

FIG. 9. DE as a function of time according to Rule (1). The two solid curves denote the DE curve corresponding to the shuffled real data. (a) The vertical bars indicate the changes of the DE curves resulting from the artificial sequences described in the text with $T$ $=8422$ and $\mu$ moving in the interval [2.094, 2.194]. (b) The vertical bars indicate the changes of the DE curves resulting from artificial sequences described in the text with $\mu=2.144$, and $T$ moving in the interval [7922, 8922].

(28). At this stage we are ready to compare the DE curves generated by the artificial data to the DE curves generated by the real data, using both Rule (1) and Rule (2). The comparison is made with the DE curves corresponding to shuffled data, since the artificial sequences are generated without correlation among the numbers $\tau_{i}$.

Let us discuss first the results concerning Rule (1). These results are illustrated in Figs. 9. In Fig. 9(a) we show the effect of changing $\mu$ in the interval [2.094,2.194], with $T$ $=8422$ and in Fig. 9(b) we show the effect of changing $T$ in the interval $[7922,8922]$, with $\mu=2.144$. We see that the DE curves of the artificial sequences fluctuate within an error strip containing the DE curve of the real data. The size of this error strip increases upon change of time and we see that the spreading caused by the change of $T$ is much smaller than that caused by the change of $\mu$. From a qualitative point of view, the results concerning Rule (2), shown in Figs. 10(a) and $10(\mathrm{~b})$, are very similar.

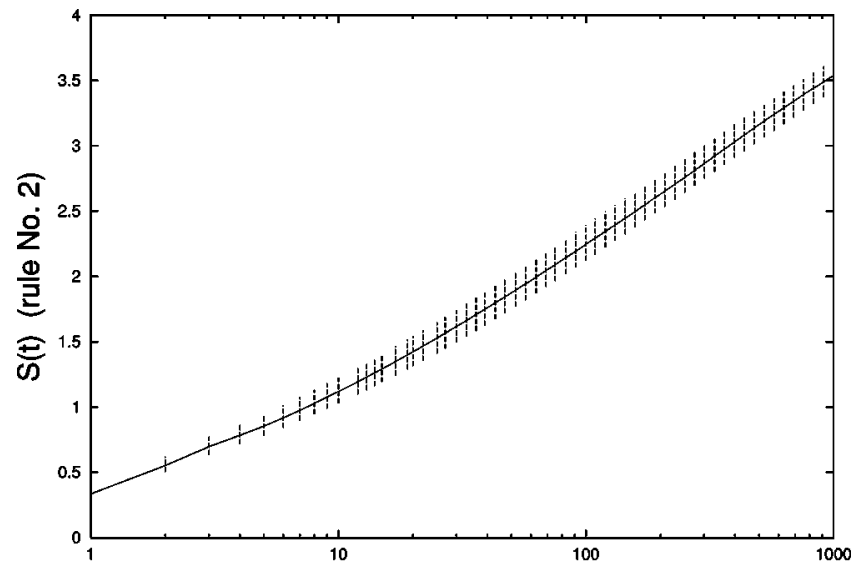

(a) $t$ (hours)

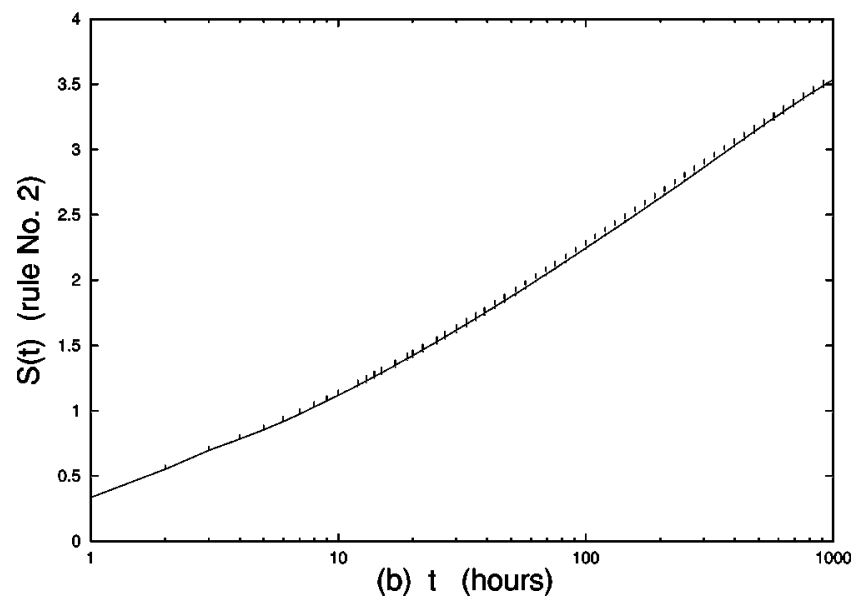

FIG. 10. DE as a function of time according to Rule (2). The two solid curves denote the DE curve corresponding to the shuffled real data. (a) The vertical bars indicate the changes of the DE curves resulting from the artificial sequences described in the text with $T$ $=8422$ and $\mu$ moving in the interval [2.094, 2.294]. (b) The vertical bars indicate the changes of the DE curves resulting from artificial sequences described in the text with $\mu=2.144$, and $T$ moving in the interval [7922, 9922].

\section{A. A more accurate measurement of $\boldsymbol{\mu}$}

We have seen that the area of the $T$-error strip is significantly smaller than that of the $\mu$-error strip, at least five times smaller. Therefore, we can improve the accuracy of $\mu$ by assigning to $T$ a fixed value and looking for the value of $\mu$ ensuring the best fitting of the real data. We assign to $T$ the value of 8422 , and we proceed with the search for the best fitting. The results are illustrated in Figs. 11(a) and 11(b). The result concerning Rule (1) is good, as seen in Fig. 11(a). As expected, Fig. 11(b) shows that the result concerning Rule (2) is even better, and we think that it can be judged to be excellent. This extremely accurate result is due to the DE curve of the artificial sequence coinciding with the DE curve of real data over the wide range of $1000 \mathrm{~h}$ of diffusion. On the basis of this excellent fitting, we conclude that

$$
\mu=2.138 \pm 0.01
$$




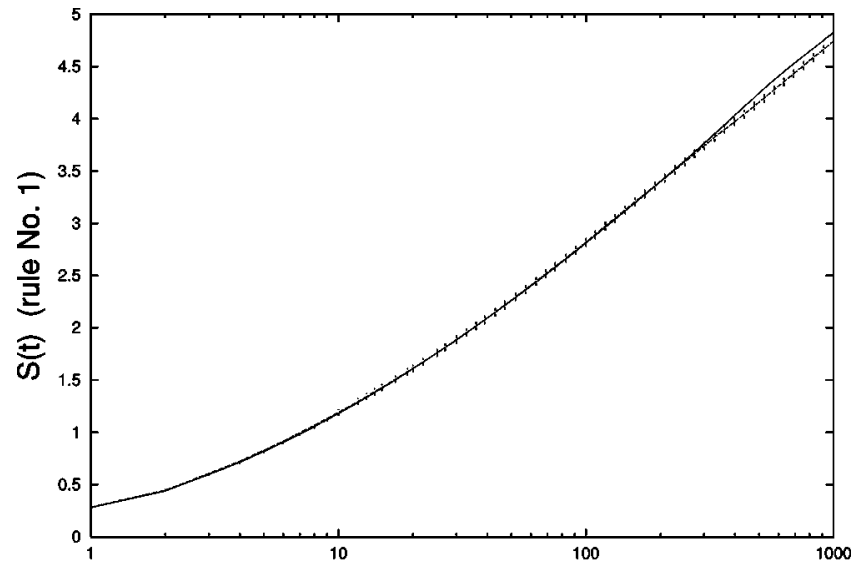

(a) $\mathrm{t}$ (hours)

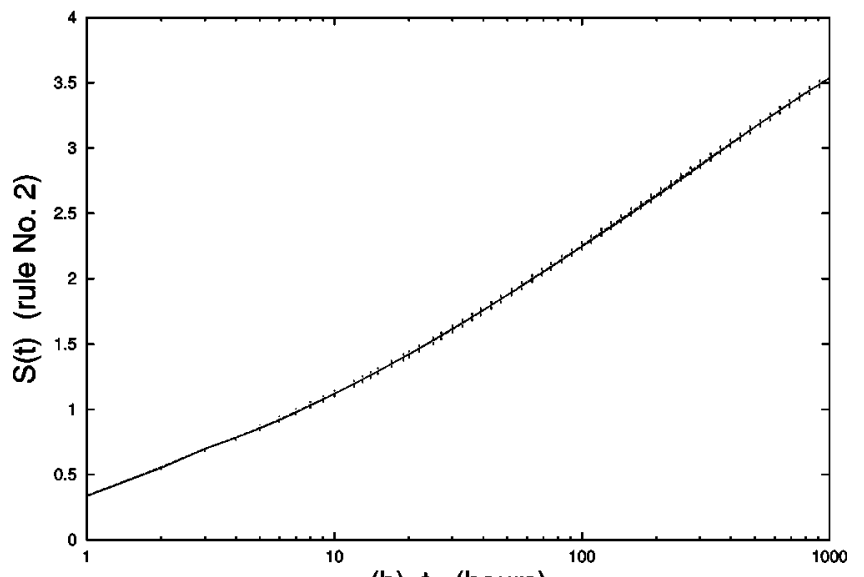

(b) $\mathrm{t}$ (hours)

FIG. 11. DE as a function of time. The solid lines denote the DE curve generated by the shuffled real data, and the dashed lines, which almost coincide with the solid lines, denote the DE curves resulting from the artificial sequence with $\mu=2.138$ and $T=8422$. (a) Rule (1). (b) Rule (2).

\section{B. Nonshuffled data and an artificial sequence with suitable memory}

In Sec. VI, we have noticed that the result of the DE analysis depends on whether the real data are shuffled or not. We think that in the original data there are signs of the 11year solar cycle and other subcycles. This makes it harder to establish a connection between the scaling $\delta$ and the power index $\mu$. However, if our conclusion that $\mu=2.138 \pm 0.01$ is correct, it should be possible to fit the DE curve of the nonshuffled original data with no further change of the fitting parameters $T$ and $\mu$, provided that we sort the artificial sequence in such a way as to mimic the solar periodicity. Rather than doing that with a model, for instance, a suitable modulation of the parameter $\lambda$ of Eq. (12), we proceed in a more direct way, according to the following procedure. Let us call $R_{i}$ and $A_{i}$ the $i$ th numbers of the real and artificial sequence used in Sec. VII A, respectively. The $i$ th number of the sorted artificial sequence is denoted by $S_{i}$. The subscript $i$ ranges from 1 to $N$. The number $S_{1}$ is fixed by selecting from the set of $A_{i}$ 's the number that is closest to $R_{1}$, this being, let us say $A_{j(1)}$. We thus set $S_{1}=A_{j(1)}$. The number $A_{j(1)}$ is eliminated from the artificial sequence. Then, we

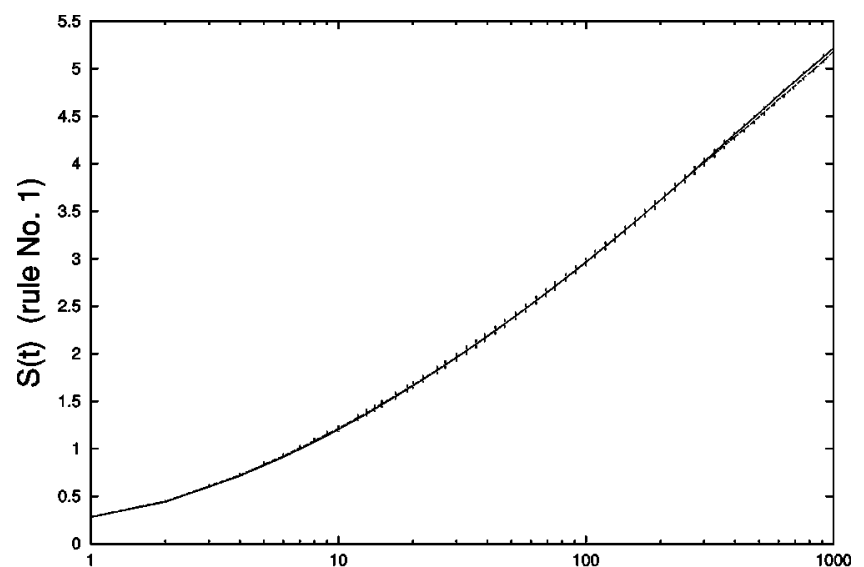

(a) $\mathrm{t}$ (hours)

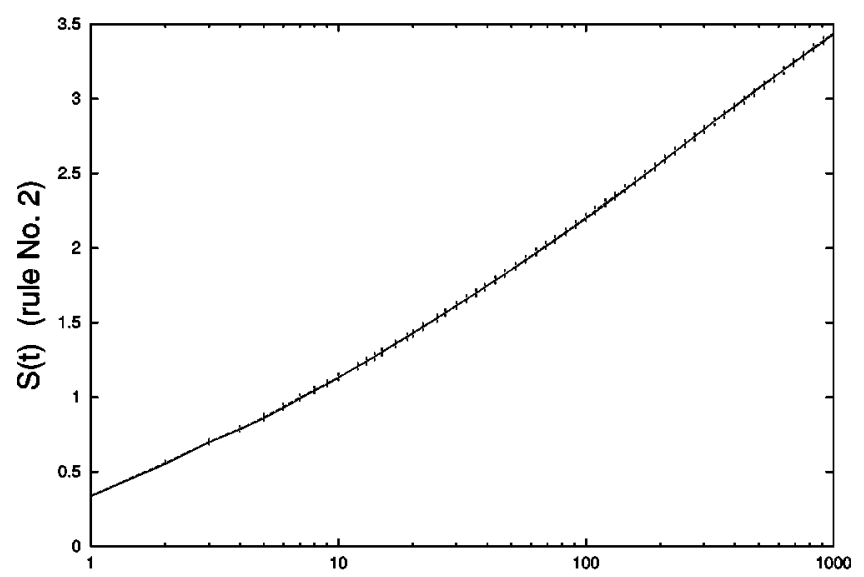

(b) $\mathrm{t}$ (hours)

FIG. 12. DE as a function of time. The solid lines denote the DE curve generated by the unshuffled real data, and the dashed lines, which almost coincide with the solid lines, denote the DE curves resulting from the artificial sequence with $\mu=2.138$ and $T=8422$ with a modulation mimicking the influence of the 11-yr solar cycle. (a) Rule (1). (b) Rule (2).

move to $R_{2}$ and from the set of the remaining $N-1$ numbers of the artificial sequence we select the closest one to it, this being, let us say $A_{j(2)}$. We proceed with the same criterion until we exhaust all the numbers of the artificial sequence. It is evident that the adoption of this procedure assigns to the artificial data a time order reflecting the complex dynamics illustrated by Figs. 2 and 3 .

At this stage, we evaluate the corresponding DE curve and we compare it to the DE curve generated by the nonshuffled real data. As earlier mentioned, the sorted artificial data are the same as those used to produce the excellent fitting of the DE curves derived from the shuffled original data. Thus, the fitting parameters are the same as those used for Figs. 11. We illustrate the result in Figs. 12, which show that the fitting accuracy is as good as [and for Rule (1) even slightly better than] the fitting of Figs. 11. This is a very remarkable result since Figs. 7 and 8 show that shuffling the data produces a significant effect. Thus, Figs. 11 and 12 prove that the memory of the data is totally under our control. 


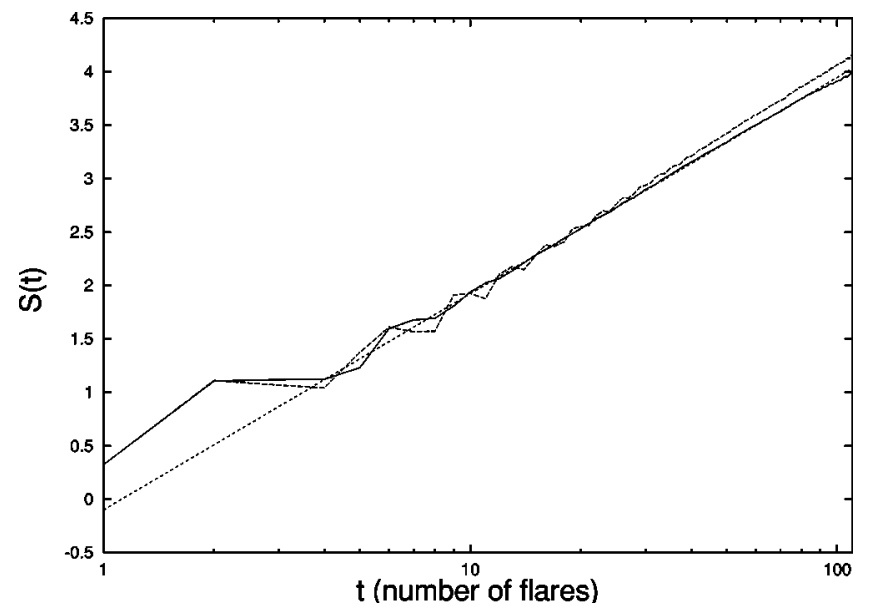

FIG. 13. DE as a function of time, according to Rule (3). The solid lines denote the DE curve generated by the shuffled real data. The dotted straight line illustrates the slope of entropy increase, $\delta$ $=0.879$, which corresponds to $\mu=2.138$. The dashed line denotes the DE curve resulting from the unshuffled real data. Note the superdiffusion of the unshuffled real data DE due to the memory in the original signal.

\section{Third rule in action}

According to Lepreti, Carbone, and Veltri [23] the waiting-time distribution $\psi(\tau)$ is already Lévy. This would imply that the adoption of the third rule yields an infinitely fast transition from dynamics to thermodynamics. This is so because Lévy distribution is stable and the convolution between two distinct Lévy distributions is a Lévy distribution [16]. According to our analysis, $\psi(\tau)$ is a shifted inverse power law. It is plausible that the difference between the shifted power-law distribution of Fig. 5 and the Lévy distribution of Ref. [23] is small. Consequently, the transition to thermodynamics is expected to be very fast. This expectation is confirmed by the numerical results illustrated in Fig. 13. The transition to the scaling regime is so fast that it is possible to detect a wide regime of linear dependence of the entropy on $\log l$, which allows us to derive for $\mu$ the value $\mu=2.138$, in total agreement with the conclusion of the earlier analysis done by means of Rules (1) and (2). We see that in this case the memory of the nonshuffled data yields a $\delta$ slightly larger than the scaling parameter of the shuffled data. The adoption of Rule (3) implies a statistical accuracy smaller than that of the other two rules, due to fact there is no limitation to the jumps intensities, thereby decreasing the number of particles located in the same cell. This has the effect of making the evaluation of $p_{i}$ and consequently that of the entropy less accurate. However, this disadvantage is widely compensated by the emergence of a much more extended scaling region that yields as a result a value of $\mu$ fully confirming that of the other two rules.

\section{CONCLUDING REMARKS}

We see that the uncertainty about the value of $\mu$ for solar flares has been significantly reduced. The current literature, if we give the same credit to all the authors, yields values of $\mu$ ranging from 3 to 1.7 . We provide the compelling conclusion that $\mu=2.138 \pm 0.01$. However, this is not the main result of his paper. We think that this paper shows that the DE method is a remarkably accurate technique of analysis that goes much beyond the direct evaluation of the waiting-time distribution $\psi(\tau)$. This is so because complex processes are characterized by two different kinds of memory. The memory of the first kind is the main object of the research work done in the field of the science of complexity. To make clear the nature of this kind of memory, let us recall [24] that a Markov master equation, namely, a stochastic process without memory, is characterized by a waiting-time distribution $\psi(\tau)$ with an exponential form, thereby implying memory for a marked deviation from the exponential condition. This is why the search for an inverse power-law distribution with a finite value of $\mu$ (the exponential distribution means $\mu$ $=\infty)$ can be interpreted as a search for memory. This is the memory of the first kind, to which the prescriptions of Ref. [9] are referred. For real data, in addition to this form of memory, another type of memory might be present, denoted by us as memory of the second type, under the form of correlation among the values $\tau_{i}$. In this paper we have seen that this second form of memory is given, in this case, by the $11-y r$ solar periodicity. It is possible that this form of additional memory is present in many other complex processes for different reasons. It is also evident that it is difficult, or perhaps impossible to reveal this form of additional memory by means of the direct evaluation of $\psi(\tau)$. This paper proves that the joint use of the direct evaluation of $\psi(\tau)$ [or of $\Psi(\tau)]$ and of the DE method is a very useful supplement to the ordinary technique, and that it can be profitably used to shed light on the dynamics behind the time series generated by complex processes.

This paper yields a convincing conclusion concerning the distinction between two possible forms of nonstationary behavior. As pointed out in Sec. III, the claim that the waitingtime distribution $\psi(\tau)$ has the form of Eq. (11) is equivalent to assuming that the dynamics of the flaring process is driven by the model of Eq. (12) with the assumption that the trajectories are injected back randomly. This is a stationary model that in the case where $z>2 \quad(\mu>2)$, would be incompatible with the existence of an invariant distribution [7] and consequently with "thermodynamic equilibrium." The inaccuracy of the analyses done by earlier work in this field would prevent us from distinguishing this form of nonstationary behavior from a genuine form of nonstationary behavior. By genuinely nonstationary behavior, we mean the existence of rules changing with time. This form of genuinely nonstationary behavior might be modeled, for instance, by assuming that the parameter $\lambda$ of Eq. (12) is time dependent. If we make the assumption that the time dependence of $\lambda$ has a period of 11 years, and we make our analysis over a period of time, that is, not much larger than this time period, as we have done, then the process must be perceived as being genuinely nonstationary. Our analysis is so accurate as to rule out the former form of nonstationary behavior and to detect significant effects stemming from the latter, or, equivalently, from the existence of the memory of the second type.

The original motivation for this paper has been to show in 
action the method of entropic analysis recently developed by our group [8-10], so as to afford indisputable results that might help the researcher of this field to establish whether the proponents of self-organized criticality [25] or those of turbulence $[3,4]$ are right. Let us try to reach a conclusion on this issue, on the basis of the results of this paper. The dynamical model of Sec. III is inspired by the models of turbulence, but we mainly use it to generate artificial sequences mimicking the real ones with no claim that it is an exhaustive picture of the dynamics behind solar flares. The fitting of Fig. 6 seems as good as the fitting of Fig. 1 of Ref. [23]. However, our analysis does not rest only on the waiting-time distribution. In a very recent paper Wheatland [26] criticized the work of Ref. [23] as being based on the assumption that the rate of solar flares is constant. This is not so, as shown by Fig. 3. On the other hand, modeling the time dependence of this rate is not easy, since it does not correspond only to a 11 -yr periodic motion but to a much more complex condition, as illustrated in Fig. 4. In fact, this figure shows that there are many other components in action. This is the reason why we decided to mimic the time dependence of the solar flare rate sorting the artificial sequence in the way described in Sec. VII B. We found that this yields a fitting with the real data as good as the fitting between the DE curve produced by the artificial sequence, with no sorting induced memory, and the DE curve produced by the shuffled real data. This is, in our opinion, a strong indication that the value of $\mu=2.138$ is a genuine property of real data. In principle, this compelling conclusion would not rule out the adoption of a modulated Poisson process of the same kind as that advocated by Wheatland [26]. This is so because, as shown in Ref. [27], the time modulation of a Poisson process can also yield $\mu$ $<3$ as well as $\mu=3$, as in the work of Wheatland [26]. In other words, using the terminology adopted in this paper, we cannot rule out the possibility that only memory of the second type is responsible for $\mu=2.138$. On the other hand, Lepreti et al. [23] applied a test for local Poisson hypothesis and they claim that this proves a departure of flare statistics from a local Poisson distribution. Under this condition it seems to be difficult to derive $\mu=2.138$ from a local Poisson statistics with the modulation produced by the solar cycle.

In our notation, the power index found by the authors of Ref. [23] is $\mu=2.38$, a value that turns out to be compatible with the uncertainty interval associated with the determination of $\mu$ by means of the direct evaluation of $\psi(\tau)$. Our analysis establishes a connection with Lévy statistics, in accordance again with the conclusions of Ref. [23]. However, we adopt a perspective that is different from that of the authors of Ref. [23]. Our diffusion process reaches the Lévy regime after the process of transition from dynamics to thermodynamics that has been discussed in detail in the earlier sections. This process is very fast if Rule (3) is adopted, but it is not infinitely fast as in the perspective of the authors of Ref. [23], who assume the waiting-time distribution $\psi(\tau)$ to obey already the Lévy statistics. We do not rule out the possibility that $\psi(\tau)$ is a stretched exponential [19]. In fact, a stretched exponential would not conflict with the attainment of Lévy statistics in the long-time limit of the diffusion process. Although a truncation of $\psi(\tau)$ at large values of $\tau$ generates a finite second moment, and consequently Gaussian statistics in the long-time limit, the transition to the conventional thermodynamic regime is ultraslow [20]. It is known [21] that a much earlier transition to Lévy statistics occurs and that the Lévy regime lasts for a very extended period of time. The transition to the Gaussian regime probably takes place at times much larger than the saturation time, and might be made visible only in the ideal case of infinitely large sequences.

In conclusion, with all the earlier warnings in mind, we can conclude that the statistical analysis of this paper lends support to the turbulence perspective. We hope furthermore that this paper affords a criterion of analysis that might help to settle not only that of solar flares, but also other delicate issues concerning complex systems.

\section{ACKNOWLEDGMENT}

We thank the BATSE/CGRO team, NASA/Goddard Space Center, Greenbelt, Maryland, for generously providing the data.
[1] E. N. Parker, Astrophys. J. 330, 474 (1988); Sol. Phys. 121, 271 (1989).

[2] R. P. Lin, R. A. Schwarz, S. R. Kane, R. M. Pelling, and K. C. Hurley, Astrophys. J. 283, 421 (1984); S. Sturrock, P. Kaufmann, P. L. Moore, and D. F. Smith, Sol. Phys. 94, 341 (1984); N. B. Crosby, M. J. Aschwanden, and B. R. Dennis, ibid. 143, 275 (1993).

[3] G. Boffetta, V. Carbone, P. Giuliani, P. Veltri, and A. Vulpiani, Phys. Rev. Lett. 83, 4662 (1999).

[4] P. Giuliani, V. Carbone, P. Veltri, G. Boffetta, and A. Vulpiani, Physica A 280, 75 (2000).

[5] M. S. Wheatland, Astrophys. J. 536, L109 (2000).

[6] M. Annunziato and P. Grigolini, Phys. Lett. A 269, 31 (2000).

[7] M. Ignaccolo, P. Grigolini, and A. Rosa, Phys. Rev. E 64, 026210 (2001).
[8] M. Scafetta, P. Hamilton, and P. Grigolini, Fractals 9, 193 (2001).

[9] P. Grigolini, L. Palatella, and G. Raffaelli, e-print cond-mat/0104166.

[10] N. Scafetta, V. Latora, and P, Grigolini, e-print cond-mat/0105041.

[11] C.-K. Peng, S. V. Buldyrev, S. Havlin, M. Simons, H. E. Stanley, and A. L. Goldberger, Phys. Rev. E 49, 1685 (1994).

[12] K. Hu, P. Ch. Ivanov, Z. Chen, P. Carpena, and H. E. Stanley, Phys. Rev. E 64, 011114 (2001).

[13] C. Beck and F. Schlögl, Thermodynamics of Chaotic Systems (Cambridge University Press, Cambridge, 1993).

[14] J. R. Dorfman, An Introduction to Chaos in Nonequilibrium Statistical Mechanics (Cambridge University Press, Cambridge, 1999). 
[15] A. I. Khinchin, Mathematical Foundations of Statistical Mechanics (Dover Publications, New York, 1949).

[16] B. V. Gnedenko and A. N. Kolmogorov, Limit Distributions for Sum of Independent Random Variables (Addison-Wesley, Reading, 1954).

[17] P. Grigolini, M. Pala, and L. Palatella, Phys. Lett. A 285, 49 (2001).

[18] W. Feller, Trans. Am. Math. Soc. 67, 98 (1949).

[19] J. Laherrére and D. Sornette, Eur. Phys. J. B 2, 525 (1998).

[20] R. N. Mantegna and H. E. Stanley, Phys. Rev. Lett. 73, 2946 (1994).

[21] E. Floriani, R. Mannella, and P. Grigolini, Phys. Rev. E 52, 5910 (1995).
[22] W. H. Press, S. A. Teukolsky, W. T. Vetterling, and B. P. Flannery, Numerical Recipes in $C$ (Cambridge University Press, Cambridge, 1992).

[23] F. Lepreti, V. Carbone, and P. Veltri, Astrophys. J. 536, L133 (2001).

[24] D. Bedeaux, K. Lakatos Lindenber, and K. E. Shuler, J. Math. Phys. 12, 2166 (1971).

[25] M. S. Wheatland, P. A. Sturrock, and J. M. McTiernan, Astrophys. J. 509, 448 (1998).

[26] M. S. Wheatland, e-print astro-ph/0107147.

[27] P. Allegrini, P. Grigolini, and A. Rocco, Phys. Lett. A 233, 309 (1997). 